\title{
Planos de manejo das unidades de conservação em pesquisas cientificas: uma forma de aproximação sociedade-universidade
}

Há uma demanda por pesquisas e informações que sustentem o planejamento e as práticas em áreas protegidas. Paralelamente, existe o compromisso socia histórico das universidades brasileiras de extensão do ensino e pesquisa à sociedade. Considerando essas interfaces, o presente trabalho tem como objetivo investigar como os Planos de Manejo são abordados nas pesquisas científicas publicadas em artigos. Para isso foi realizado procedimento de revisão da literatura. Os resultados são advindos de 39 artigos selecionados dos quais $25,64 \%$ tratam de aspectos de gestão e governança das áreas. Apenas quatro artigos tiveram como objeto de estudo o Plano de Manejo. O Plano de Manejo serve como referência bibliográfica em 30,77\% dos artigos analisados, revelando que são menores as investigações que têm o Plano de Manejo com maior ênfase na pesquisa. Apesar de a maior parte dos artigos encontrados indicarem que podem contribuir com a elaboração dos Planos de Manejo locais e discutirem sobre as fraquezas dos Planos vigentes nas UCs investigadas, somente $11,11 \%$ destes centram-se em discutir os Planos de Manejo, colaborando assim com as UCs em questão. Nesse sentido, verifica-se a distância entre academia e ações sociais. Conclui-se que há a necessidade de publicação de resultados revisados por pares para construção do conhecimento dessas áreas, especialmente para avaliação e melhora do Plano de Manejo enquanto ferramenta que promova e garanta manejo efetivo e gestão participativa. Ademais, recomenda-se que pesquisadores se coloquem à disposição para colaboração real e prática nos locais que lhes são objeto de estudo. Da mesma forma, faz-se necessário que as comunidades busquem apoio das universidades.

Palavras-chave: Plano de Manejo; Áreas Protegidas; Gestão; Extensão Universitária.

\section{Protected areas' management plans in scientific research: a pathway towards society-academy approximation}

There is a demand for research and information to support planning and practices in protected areas. At the same time, there is a social and historic commitment from Brazilian universities (major research centers in the country) to extend research and knowledge towards the community. Considering these interfaces, this literature review aims to research how management plans are presented in scientific papers. The review resulted in a collection of 39 papers in which $25.64 \%$ discussed management and governance aspects. Only four papers had management plans as the research's focal point. For $30.77 \%$ the management plan was listed as a reference implying that there are few researches that put management plans with a bigger emphasis on the research. Although the majority of papers cite that their research can contribute to the elaboration and review of the management plan, only $11.11 \%$ effectively focus on discussing the plan. We conclude that more scientific peer reviewed results need to be published to build the knowledge about this instrument and effective management. Furthermore, we recommend that researchers make themselves available for real and practical collaboration in the protected areas that are the object of study.

Keywords: Management Plan; Protected Areas; Management; University Extension.

Topic: Planejamento, Gestão e Políticas Públicas Ambientais

Reviewed anonymously in the process of blind peer
Received: 06/07/2021

Approved: $27 / 07 / 2021$

Bruna Lima Ferreira (iD)

Universidade de São Paulo, Brasil

http://lattes.cnpq.br/5524904680933378

https://orcid.org/0000-0002-7673-7733

bruna04lima@gmail.com

Suzana Rodrigues de Araujo (iD)

Universidade de São Paulo, Brasil

http://lattes.cnpq.br/8739343300791997

http://orcid.org/0000-0001-6026-4671

suroaraujo@gmail.com

\section{Mayra Antonelli Ponti (iD)}

Universidade de São Paulo, Brasil

http://lattes.cnpq.br/3579795353029919

http://orcid.org/0000-0003-4931-2788

antonelli.may@gmail.com



DOI: 10.6008/CBPC2179-6858.2021.007.0043

Referencing this:

FERREIRA, B. L.; ARAUJO, S. R.; PONTI, M. A.. Planos de manejo das unidades de conservação em pesquisas científicas: uma forma de aproximação sociedade-universidade. Revista lbero Americana de Ciências Ambientais, v.12, n.7, p.497-510, 2021. DOI: http://doi.org/10.6008/CBPC2179-6858.2021.007.0043 


\section{INTRODUÇÃO}

A estratégia de delimitação de áreas protegidas para proteção ambiental é fundamental para a conservação da natureza (OLDEKOP et al., 2015; GELDMANN et al., 2013; BUTCHART et al., 2012). Porém, estas áreas possuem potencial para além da conservação, englobando elementos econômicos e sociais (WATSON et al., 2014) e contribuindo para diferentes objetivos de desenvolvimento sustentável, como a mitigação dos impactos das mudanças climáticas (BEBBER et al., 2017).

Todo o potencial que as áreas protegidas apresentam para proteção ambiental não é alcançado apenas pela criação ou extensão de suas coberturas. $O$ impacto positivo social, econômico e na conservação ambiental depende de um manejo efetivo destas áreas (GILL et al., 2017; OLDEKOP et al., 2015; WATSON et al., 2014; GELDMANN et al., 2015) e, consequentemente, de sua boa governança, condição para que este manejo ocorra (LOCKWOOD, 2010; MACURA et al., 2015). Avaliações da efetividade no manejo se sucedem de forma a verificar se os objetivos e valores vinculados a área protegida estão sendo alcançados (HOCKINGS et al., 2006) e fazem parte do padrão global de inclusão de áreas protegidas na Lista Verde da União Internacional para a Conservação da Natureza (IUCN et al., 2019). Assim, um manejo efetivo passa por processos de planejamento nos quais são delineados objetivos e estratégias para conservação da área (HOCKINGS et al., 2006).

No Brasil, há o Sistema de Unidades de Conservação (SNUC, Lei 9985 de 2000). Um sistema de áreas protegidas, conhecidas como Unidades de Conservação (UCs), divididas em dois grupos: Unidades de Proteção Integral e Unidades de Uso Sustentável (BRASIL, 2000). O primeiro grupo abrange categorias de manejo mais restritivas ao uso; enquanto o segundo permite o uso direto, desde que sustentável, dos recursos naturais. Este sistema estabelece que as UCs tenham um planejamento do manejo da área definido em documento técnico que delineie objetivos e estratégias para conservação ambiental nestes espaços e de acordo com a categoria que pertencem. Tal documento é conhecido como Plano de Manejo (BRASIL, 2000). O Plano de Manejo abrange a manutenção e gestão das UCs e é reconhecido nacional e internacionalmente (ICMBio, 2018). É um documento importante não só para garantir o manejo da área, mas contempla também princípios de governança ao incluir em sua elaboração e revisão o incentivo à participação de diferentes atores sociais, dentre eles as instituições de ensino e pesquisa (BRASIL, 2017; ICMBio, 2018). A participação de instituições de pesquisa é essencial e encorajada (Artigo 4 e 5, BRASIL, 2000), já que este documento depende de estudos detalhados dos fatores físicos e biológicos da área (MAGANHOTTO et al., 2014), bem como de informações técnico-científicas e saberes tradicionais acerca da região (BRASIL, 2017; ICMBio, 2018).

O rigor metodológico de uma pesquisa científica pode trazer resultados mais precisos sobre a área e, assim, auxiliar na tomada de decisão, planejamento e monitoramento de estratégias. Por exemplo, por meio da construção de indicadores de capacidade de carga (WAGAR, 1974), avaliação de desafios do turismo nestas áreas, desenvolvimento do inventário de fauna (BOLLER et al., 2020) e flora (MESSIAS et al., 2017) e identificação de problemas e suas possíveis soluções (CANTO et al., 2020; BERTOLIN, 2020). Estes fatores, 
somados a delineamentos de articulação entre a UC e a comunidade científica (Artigo 32, BRASIL, 2000) evidenciam a importância de parcerias destas áreas com instituições de pesquisa, como as universidades, que são grandes contribuintes para ciência no país.

Há uma demanda por pesquisas e informações que sustentem o planejamento e prática em áreas protegidas. Paralelamente, existe o compromisso social histórico das universidades brasileiras de extensão do ensino e pesquisa à sociedade (SANTOS, 2012; SILVA, 2013; ANTONELLI-PONTI et al., 2018). Este compromisso está previsto na legislação como uma das finalidades da Educação Superior brasileira (BRASIL, 1996). Dessa forma, cientistas vinculados a universidades têm o dever, não só ético, mas também social, de comunicar aos atores locais pesquisas realizadas em UCs. Outro ponto importante é que com os avanços tecnológicos e a facilidade crescente do acesso à informação, a comunicação científica se aproximou da divulgação científica, sendo possível que o acesso a artigos acadêmicos, por exemplo, seja feito para além da comunidade acadêmica (VALÉRIO et al., 2008), incluindo gestores de UCs e outros responsáveis pelo manejo e boa governança dessas áreas. Cabe ainda ressaltar o papel da comunicação científica para construção do conhecimento (VALÉRIO et al., 2008), permitindo que outros cientistas tenham acesso a pesquisas anteriores da área e ajustem, adicionem e verifiquem as mesmas. A pesquisa científica é uma base importante para o planejamento das áreas protegidas brasileiras, como as Unidades de Conservação por meio do instrumento do Plano de Manejo. Nesse contexto, pressupõe-se que a comunicação científica dos trabalhos realizados em UCs tem potencial para auxiliar e complementar o conhecimento que se tem sobre essas áreas de proteção, sua eficácia para conservação e impactos para o desenvolvimento sustentável regional e nacional auxiliando, assim, na construção de uma base de dados mais robusta para elaboração e revisão dos Planos de Manejo.

Considerando essas interfaces, o presente trabalho tem como objetivo investigar como os Planos de Manejo são abordados nas pesquisas científicas publicadas em artigos. Buscamos, ainda, fazer uma caracterização temporal, geográfica e por categoria de UCs destes artigos, identificando quais são as áreas do conhecimento que mais citam este tipo de documento em seus estudos. Ainda, devido à importância do plano para o manejo efetivo das UCs, quantificamos, por meio de uma revisão bibliográfica, as pesquisas que trazem este instrumento como objeto central do estudo.

\section{METODOLOGIA}

A revisão bibliográfica teve como base os componentes indicados no checklist PRISMA - P (SHAMSEER et al., 2015) e nos artigos de Sousa et al. (2018) e Khan et al. (2003). O levantamento bibliográfico foi realizado por dois revisores, que buscaram e leram os artigos, e um supervisor neutro, que realizou a verificação geral dos dados, etapas e resultados, evitando riscos de viés de pesquisa e de interpretação.

Considerou-se na revisão apenas artigos científicos revisados por pares, pois estes são os principais meios de comunicação científica, nos idiomas português, inglês e espanhol. Buscou-se por artigos publicados entre o ano 2000 (ano em que se estabeleceu o Sistema Nacional de Unidades de Conservação, Lei 9985 de 2000) até novembro de 2020. Não foram incluídas as referências bibliográficas citadas nos artigos. Apenas artigos que citavam o Plano de Manejo e Unidade de Conservação já em seu resumo, palavras-chave ou título 
foram considerados para esta seleção.

A primeira etapa da revisão se baseou em uma varredura dos artigos disponíveis online. Neste momento, dois revisores, de forma independente, realizaram a pesquisa nas plataformas de busca: Periódicos CAPES, Scielo e Scopus, usando as palavras chave: 'Plano de Manejo' e 'Unidade de Conservação' ou 'Management Plan' e 'Protected Areas'. O número total de artigos encontrados foi registrado. Cada revisor então utilizou os critérios de inclusão elencados e os artigos selecionados foram salvos em uma pasta com o nome do arquivo padronizado.

O segundo momento de varredura aconteceu a partir da análise conjunta dos revisores, que compararam os artigos selecionados na etapa anterior. Os artigos divergentes foram reavaliados e, em comum acordo, descartados ou inclusos.

Após estas etapas, todos os artigos foram lidos buscando responder às seguintes questões: I. É um artigo de revisão ou discussão teórica? II. O Plano de Manejo da Unidade de Conservação é objeto de estudo? - Se sim, qual aspecto do Plano foi analisado? - Caso não seja o tema central, em qual etapa do artigo ele aparece (introdução, metodologia, resultados)? - Caso não seja o tema central, qual é? III. Qual a categoria de Unidade de Conservação mencionada no artigo? IV. Qual local (estado ou região) do Brasil o artigo aborda? V. Quais as palavras-chave selecionadas pelos autores do artigo? VI. Em qual grande tema ou área do conhecimento o artigo se enquadra?

Cabe ressaltar que a leitura e análise dos artigos foi realizada de forma independente pelos dois revisores. Assim, um segundo momento de comparação de dados foi realizado. Artigos que não se enquadraram nos critérios de inclusão e passaram pelas primeiras varreduras foram excluídos e as respostas às perguntas foram comparadas e discutidas. Todas estas etapas foram previamente aprovadas pelo terceiro revisor neutro.

Para avaliar a forma como plano de manejo é abordado nos artigos foi feita uma análise do contexto em que o termo é citado no resumo e em quais locais dos textos (introdução, metodologia, resultado e discussão e considerações finais) o termo foi citado.

As palavras-chave dos artigos, juntamente com seus objetivos, foram utilizadas para criação de categorias para classificação das publicações em diferentes áreas de pesquisa. O processo foi indutivo, o qual visa a compreensão dos objetos de pesquisa a partir do conteúdo investigado (BARDIN, 1991) e partiu de uma categorização mais específica até generalizações maiores para produzir as doze categorias finais. Um revisor fez a primeira sistematização e descrição de categorias e, posteriormente, os outros revisores classificaram os trabalhos dentro do sistema. A concordância da classificação foi feita por meio do coeficiente de concordância de Kappa (0,85, índice de concordância de 86,32\%). As categorizações inicialmente discordantes foram discutidas e um acordo para alocação final dos artigos em categorias foi alcançado.

\section{DISCUSSÃO TEÓRICA}

Na primeira etapa de varredura, a Plataforma de Periódico Capes foi a que mais gerou resultados (303), dos quais apenas $6,27 \%$ foram selecionados para revisão. Um número menor de resultados foi obtido 
nas buscas nas plataformas Scielo (24 resultados) e Scopus (15 resultados), porém com percentual de aproveitamento maior, $83,33 \%$ e $20 \%$ de artigos selecionados, respectivamente. Na segunda etapa, verificamos que havia artigos repetidos na Plataforma de Periódicos Capes e Scielo, assim, de um total de 42 artigos selecionados, dois foram excluídos. Após a leitura direcionada dos trabalhos, mais um artigo foi retirado por não dizer respeito ao Plano de Manejo de Unidade de Conservação. Dessa forma, a base de artigos levantados nesta revisão sistemática foi de 39 publicações.

Apenas quatro artigos têm como objeto de estudo o Plano de Manejo. No restante, uma análise dos resumos indica que a maioria $(46,15 \%)$ levanta seus resultados como uma contribuição para elaboração de plano ou faz críticas aos planos das unidades de conservação estudadas. Destes, apenas dois artigos evidenciam que fazem parte de uma iniciativa de revisão ou elaboração de Plano de Manejo. Ao analisar a citação do termo 'Plano de Manejo' ao longo de todo texto do artigo, apenas cinco estudos (dois dos que analisam o plano como objeto de estudo) citam o termo ao longo de todas as etapas do artigo: introdução, metodologia, resultados, discussão e conclusão.

A maior parte dos trabalhos (48,72\%) foi realizada em Parques, $43,58 \%$ no Sudeste e as publicações têm picos máximos de cinco artigos nos anos 2014 e 2018. No geral, os artigos se encaixam em 12 diferentes áreas de estudos, sendo que a maioria $(43,59 \%)$ está classificada como estudos de gestão e governança ou geologia e geomorfologia.

\section{Caracterização geral dos artigos levantados}

Não há artigos publicados entre os anos de 2000 e 2003 ou entre 2006 e 2008 que contemplem os critérios de elegibilidade descritos neste trabalho. Nota-se um crescimento na quantidade de artigos que abordam os temas Plano de Manejo e Unidade de Conservação a partir de 2004, mas percebe-se instabilidade na quantidade deste tipo de publicação ao longo do tempo (Figura 1).

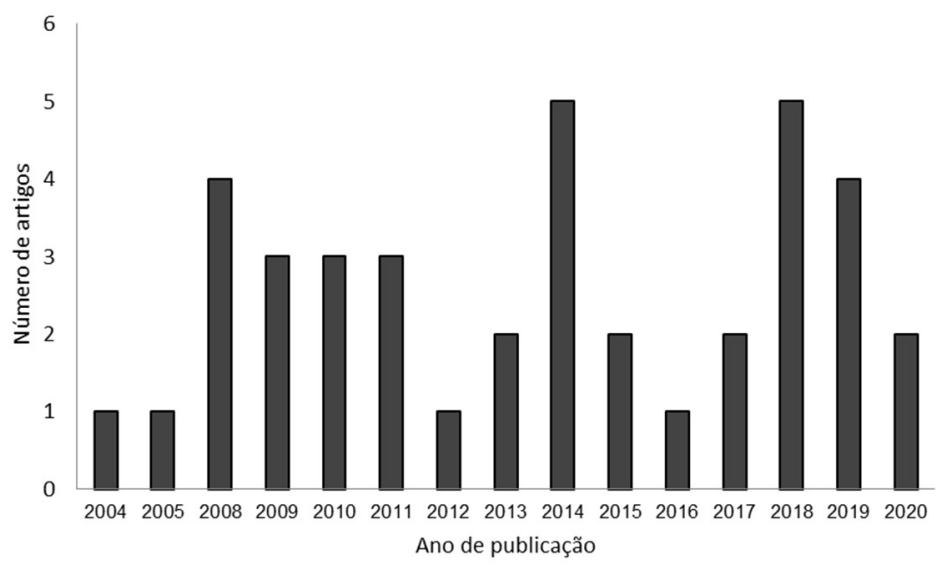

Figura 1: Número de artigos publicados no período entre 2004 e 2020.

Nos períodos de 2008 a 2011, 2014, 2018 e 2019 houve três ou mais publicações, apenas 2014 e 2018 com um pico de cinco artigos. A irregularidade na tendência de número de artigos no decorrer dos anos pode refletir a irregularidade da própria elaboração dos Planos de Manejo. Em 2019, apenas 19,05\% das Unidades de Conservação possuem esse documento, sendo que o prazo para elaboração do Plano de Manejo após 
criação da UC é de cinco anos (BRASIL, 2000).

É difícil estipular uma causa para a distribuição dos artigos nos anos, porém, alguns acontecimentos e dados podem ter alguma influência em estudos realizados em UCs e outras áreas protegidas. Considerando um prazo médio de dois anos entre a submissão e publicação de um artigo científico, os períodos com mais de três artigos publicados referentes ao tema podem se relacionar com o aumento do foco na importância das áreas protegidas para conservação. Por exemplo, o acordo internacional do Plano Estratégico de Biodiversidade assinado na 10ạ Conferência das Partes da Convenção sobre Diversidade Biológica (COP-10), em 2011, que visava metas para aumento de áreas protegidas para conservação até 2020, a Conferência das Nações Unidas sobre Desenvolvimento Sustentável, em 2012, que resultou nos Objetivos de Desenvolvimento Sustentável da ONU e, no contexto brasileiro, o novo Código Florestal (BRASIL, 2012), que pode ter gerado um aumento no interesse pela área.

Além disso, em 2018 houve um aumento na área protegida no país, subindo de 1.263,55 km² para $925.209,38 \mathrm{~km}^{2}$, o maior aumento desde 2000 e pico no número de áreas protegidas criadas (97 UCs, atrás apenas dos anos de 2001, 2002, 2008 e 2010 nos quais foram criadas mais de 100 UCs.

Nos artigos levantados nesta revisão, as regiões que possuem maior número de pesquisas são: Sudeste $(43,58 \%)$ e Nordeste (23,08\%). A Tabela 1 sistematiza a distribuição de artigos pelos estados e regiões. É importante ressaltar que um artigo trabalhou com áreas localizadas nos estados do Piauí, Ceará e Pernambuco.

Tabela 1: Número de artigos de acordo com região e estado aos quais a(s) Unidade(s) de Conservação estudada(s) se localiza $(m)$.

\begin{tabular}{|l|l|l|}
\hline Região & Estado & Número de artigos \\
\hline \multirow{4}{*}{ Sudeste } & São Paulo & 7 \\
\cline { 2 - 3 } & Minas Gerais & 7 \\
\cline { 2 - 3 } & Rio de Janeiro & 3 \\
\hline \multirow{5}{*}{ Norte } & Rondônia & 1 \\
\cline { 2 - 3 } & Pará & 1 \\
\cline { 2 - 3 } & Amazonas & 2 \\
\hline \multirow{5}{*}{ Sul } & Bahia & 4 \\
\cline { 2 - 3 } & Pernambuco & 2 \\
\cline { 2 - 3 } & Ceará & 1 \\
\cline { 2 - 3 } & Piauí & 2 \\
\cline { 2 - 3 } & Rio Grande do Norte & 1 \\
\cline { 2 - 3 } & Paraíba & 2 \\
\hline Centro - Oeste & Rio Grande do Sul & 1 \\
\hline & Paraná & 3 \\
\hline Brasil & Mato Grosso do Sul & 1 \\
\hline
\end{tabular}

A região sudeste é conhecida por ser um dos grandes polos de pesquisa do país. Além disso, os estados do Rio de Janeiro, São Paulo, Minas Gerais e Espírito Santo estão entre os seis estados com maior número de UCs. Quanto ao número de trabalhos no Nordeste, a maior área preservada se concentra nos estados de Pernambuco, Paraíba e Maranhão $\left(431.950,77 \mathrm{~km}^{2}\right)$, o que pode ser um componente para esta região ser a segunda com maior número de artigos.

Além da distribuição temporal e regional das pesquisas, identificamos quais os grupos e categorias de UCs mais estudados. No Sistema Nacional de Unidades de Conservação (SNUC) existem doze categorias 
de UCs, sendo sete de Uso Sustentável e cinco de Proteção Integral. Nos artigos selecionados, foram abordadas sete destas categorias, sendo elas: duas do grupo de UCs de Proteção Integral (Estação Ecológica e Parques), além de cinco do grupo de UCs de Uso Sustentável (Reserva de Desenvolvimento Sustentável, Reserva Extrativista, Reserva Particular de Patrimônio Natural, Floresta Nacional e Área de Proteção Ambiental, Tabela 2). Um dos artigos aborda duas categorias (Parque e Área de Proteção Ambiental), portanto, foi contabilizado em ambas as classificações.

Tabela 2: Número de artigos de acordo com categorias de Unidade(s) de Conservação estudada(s). A categoria 'Não especificada' se refere a quatro artigos que indicaram Unidades de Conservação no geral e um que estudou UCs de Proteção integral sem especificar uma categoria específica.

\begin{tabular}{|l|l|l|}
\hline Grupo de Unidades de Conservação & Categorias de Unidades de Conservação & Número de artigos \\
\hline Unidade de Conservação de Proteção Integral & Estação Ecológica & 2 \\
\cline { 2 - 3 } & Parque & 19 \\
\hline \multirow{4}{*}{ Unidade de Conservação de Uso Sustentável } & Reserva de Desenvolvimento Sustentável & 1 \\
\cline { 2 - 3 } & Reserva Extrativista & 1 \\
\cline { 2 - 3 } & Reserva Particular do Patrimônio Natural & 1 \\
\cline { 2 - 3 } & Floresta Nacional & 8 \\
\cline { 2 - 3 } & Área de Proteção Ambiental & 4 \\
\hline Não especificada & Não especificada & 4 \\
\hline
\end{tabular}

Contabilizou-se que 52,5\% dos artigos selecionados estudam categorias de UCs de Proteção Integral (sendo destes, 90,48\% em Parques) enquanto 37,5\% se referem às Unidades de Uso Sustentável (sendo a Floresta Nacional a segunda categoria mais citada). Porém, há uma maior diversidade de categorias estudadas para Uso Sustentável, enquanto que para Proteção Integral a maior parte dos trabalhos se refere a Parques Federais, Estaduais ou Municipais.

Essas porcentagens podem ser parcialmente explicadas pelo fato de que, das UCs com Plano de Manejo, 55,14\% pertencem ao grupo de Proteção Integral. Porém, quase todos os estudos levantados são sobre Parques (47,5\%). Este fato pode ter relação com o maior número de UCs na categoria de parques do que em outras categorias de gestão primariamente de gestão públicas. Porém, esse não é o único motivo, já que há mais que o dobro de RPPNs do que Parques e apenas um artigo trabalha com essa categoria de área protegida de gestão privada. Outro componente pode ser que os Parques tenham mais Planos de Manejo elaborados do que RPPNs, já que, dentre as UCs que possuem Plano de Manejo, 95,14\% são de gestão pública (RPPNs tem gestão privada). Além disso, os Parques cobrem a maior quilometragem de área protegida.

A ênfase aos Parques parece então cíclica: maior número de unidades classificadas nesta categoria, que pertence ao grupo mais restrito de UC, mas que permitem e possuem grande potencial para uso público e turismo. Das 10 unidades federais mais visitadas em 2019, cinco são Parques e correspondem a 62,13\% do total de visitação (ICMBio, 2020). Esse alto número de UCs nesta categoria, unido ao seu potencial de uso, podem ser importantes incentivos aos estudos nestas áreas que, por sua vez, geram mais informações base para produção de materiais e Plano de Manejo.

Os artigos selecionados nesta revisão foram classificados em 12 categorias que representam grandes temas desta pesquisa (Tabela 3), sendo que os temas mais recorrentes foram gestão e governança $(25,64 \%)$, geologia e geomorfologia $(17,95 \%)$ e uso público ou socioambiental das UCs $(15,38 \%)$. 
Tabela 3: Descrição das categorias utilizadas para classificação e distribuição dos artigos.

\begin{tabular}{|c|c|c|}
\hline Categoria & Descrição & $\begin{array}{l}\text { Número } \\
\text { de artigos }\end{array}$ \\
\hline Uso da terra & $\begin{array}{l}\text { Artigos que tem como objetivo especializar, mapear vegetação e usos da terra ou que } \\
\text { analisem conflitos de uso de paisagem em Unidades de Conservação e em seu entorno. }\end{array}$ & 4 \\
\hline Gestão e governança & $\begin{array}{l}\text { Artigos que tem como objetivo: analisar de planos de manejo, discutir processo de } \\
\text { criação e implementação de Unidade de Conservação, avaliar efetividade de manejo, } \\
\text { conselhos gestores e a gestão de recursos naturais em Unidades de Conservação. }\end{array}$ & 10 \\
\hline Estudo de fauna & $\begin{array}{l}\text { Artigos que busquem fazer inventário, monitoramento, registro, ocorrência e investigar } \\
\text { distribuição de espécies da fauna em Unidades de Conservação. }\end{array}$ & 4 \\
\hline $\begin{array}{l}\text { Geologia e } \\
\text { geomorfologia }\end{array}$ & $\begin{array}{l}\text { Artigos que busquem fazer a caracterização geológica, geoambiental e geoecológica ou } \\
\text { o diagnóstico geomorfológico, mensuração de relevos, a estruturação e criação de bases } \\
\text { cartográficas em Unidades de Conservação. }\end{array}$ & 7 \\
\hline Zoneamento ambiental & $\begin{array}{l}\text { Artigos que busquem estratégias de investigação e análise de dados para procedimentos } \\
\text { de zoneamento ambiental em Unidades de Conservação. }\end{array}$ & 1 \\
\hline $\begin{array}{l}\text { Zonas tampão, de } \\
\text { amortecimento ou do } \\
\text { entorno }\end{array}$ & $\begin{array}{l}\text { Estudos sobre a área do entorno de unidades de conservação, analisando controvérsias } \\
\text { jurídicas relacionadas a estas áreas. }\end{array}$ & 1 \\
\hline $\begin{array}{l}\text { Uso público e estudo } \\
\text { socioambiental }\end{array}$ & $\begin{array}{l}\text { Artigos que abordam temas como: turismo, conhecimento de visitantes e moradores, } \\
\text { sugestões de moradores e visitantes para viabilizar conservação, percepção ambiental, } \\
\text { envolvimento, satisfação e conhecimentos tradicionais da população local em áreas de } \\
\text { Unidades de Conservação. }\end{array}$ & 6 \\
\hline Arqueologia & Artigos que façam diagnóstico arqueológico. & 1 \\
\hline Geodiversidade & $\begin{array}{l}\text { Artigos que tenham como objetivo analisar se geodiversidade aparece nos documentos } \\
\text { do parque. }\end{array}$ & 1 \\
\hline Estudo de flora & $\begin{array}{l}\text { Artigos que busquem fazer inventário, monitoramento, registro, ocorrência e investigar } \\
\text { distribuição de espécies da flora em Unidades de Conservação. }\end{array}$ & 1 \\
\hline $\begin{array}{l}\text { Importância } \\
\text { conservação }\end{array}$ & $\begin{array}{l}\text { Estudos que façam avaliação da importância da Unidade de Conservação para } \\
\text { conservação ambiental e da biodiversidade. }\end{array}$ & 1 \\
\hline Educação ambiental & $\begin{array}{l}\text { Estudos que façam análise de atividades de educação ambiental em Unidades de } \\
\text { Conservação. }\end{array}$ & 2 \\
\hline
\end{tabular}

É provável que estes temas apareçam em maior medida porque o plano é uma ferramenta de gestão e manejo (BRASIL, 2000; ICMBio, 2018), além de ser um documento que conta com a participação pública, especialmente dos conselhos gestores (BRASIL, 2017; ICMBio, 2018). Assim, por se tratar de um documento que envolve diferentes atores sociais em sua elaboração e traz consequências diretas para o manejo e gestão da área protegida e de seu entorno, faz sentido que a maior parte dos estudos que citam este documento estejam relacionados a tópicos de governança, uso público e questões socioambientais.

Ressalta-se que a discussão sobre inclusão social na ideia de conservação ambiental é relativamente recente. Segundo Mace (2014), o início da inclusão do caráter multidimensional e complexo do aspecto social na conservação começou apenas por volta de 2010. Com a mudança na forma de se pensar e pesquisar conservação ambiental, os trabalhos que incluem avaliações de efetividade de manejo (HOCKINGS et al., 2006; IUCN et al., 2019) e boa governança (LOCKWOOD, 2010; MACURA et al., 2015), por exemplo, ainda são recentes e começaram a ganhar força nos últimos anos.

O Plano de Manejo ainda é um documento no qual o uso de mapas e diagnóstico geológico é considerado um componente dinâmico, essencial para diagnóstico da Unidade e inclusão de dados no Sistema de Informações Geográfica (SIG), sendo necessário para sua elaboração um técnico de geoprocessamento (ICMBio, 2018). Esta característica pode fomentar estudos relacionados a geologia e geomorfologia ligados aos Plano de Manejo.

Dos artigos selecionados, $12,82 \%$ se baseiam em revisões bibliográficas e análise documental. Adicionalmente, $7,69 \%$ deles tem como escopo do trabalho as UCs de forma generalizada, um aborda a 
categoria Área de Proteção Ambiental (APA) (ESTEVES et al., 2014) e um diz respeito ao Parque Nacional da Serra da Canastra (FERREIRA, 2015).

Estes trabalhos teóricos abarcam questões variadas, como as controvérsias relacionadas a zona de amortecimento (VITALLI et al., 2009), contribuições da Avaliação Ambiental Estratégica (AAE) para os Planos de Manejo (ESTEVES et al., 2014); possibilidades e limitações das UCs, discutindo, inclusive, o Plano de Manejo (MAGANHOTTO et al., 2014); caracterização da questão fundiária no Parque Nacional da Serra da Canastra (FERREIRA, 2015) e instrumentos de gestão de recursos naturais (BEZERRA et al., 2018a).

\section{Como o Plano de Manejo aparece nos artigos científicos?}

O Plano de Manejo serve como referência bibliográfica em 30,77\% dos artigos analisados (KOESTER, 2008; SOUZA et al., 2008; SALLUN et al., 2010; MEDEIROS et al., 2011; SILVA, 2013; MEZZOMO et al., 2014; BRITO, 2015; VIEIRA et al., 2017; MORAIS et al., 2018; ARAÚJO-BISSA et al., 2019, BERNARDI et al., 2020; GUERRERO et al., 2020). Destes, quatro tem o Plano de Manejo como objeto de estudo: analisando planos e componentes específicos (plano de uso público em Souza et al. (2008); conceito de geodiversidade presente no documento de Brito (2015)); pontos de conflito de uso e ocupação do solo entre APA e planos gestores (BERNARDI et al., 2020) e seu processo de elaboração (nos Parques Nacionais de Itatiaia, da Serra dos Órgãos, da Tijuca, da Serra da Bocaina e da Restinga de Jurubatiba, no estado do Rio de Janeiro em Medeiros et al. (2011)). Adicionalmente aos artigos que citam planos de manejo em suas referências, há mais um trabalho que analisa o processo de construção do Plano de Manejo no Parque Nacional de Superagui contrapondo conhecimentos tradicionais de uma comunidade caiçara aos conhecimentos científicos (RAINHO, 2014) e possibilidades e desafios deste documento (MAGANHOTTO et al., 2014).

Em uma análise do contexto da citação do Plano de Manejo nos resumos dos artigos restantes, a maioria $(35,9 \%)$ indica, em seus resumos, que espera ou tem o intuito de subsidiar ou contribuir para o Plano de Manejo. Destes, dois colocam que são parte da iniciativa de revisão ou elaboração do plano (BAETA et al., 2013, ANSILAGO et al., 2018). O restante dos artigos faz críticas ao documento (carece de um planejamento de atividades turísticas (SILVA, 2013); precisa ser reformulado considerando diversidade regional (VIEIRA et al., 2017) ou que as populações tradicionais foram invisibilizadas pelo plano, segundo Guerrero et al. (2020)); colocam o plano como característica da área estudada, ou seja, se possui ou não este documento (KOESTER, 2008; BEZERRA et al., 2018a; LOPES et al., 2019); citam o plano como resultado (LIMA et al., 2005; ABREU, 2016); ou o fazem apenas nas palavras-chave (VITALLI et al., 2009; BEZERRA et al., 2018b) ou no contexto da metodologia ('sob a perspectiva do plano' em Araújo-Bissa et al. (2019) e a percepção das pessoas sobre regras no plano, segundo Nascimento et al. (2019)) e contextualização/justificativa ou considerações finais (GONDIM et al., 2011; MEZZOMO et al., 2014; FERREIRA, 2015; SOUZA et al., 2018; CAMPOS et al., 2019).

Ao analisar a distribuição do termo 'Plano de Manejo' ao longo de todo o texto do artigo, apenas cinco estudos o citam em todas as seções do artigo (na introdução, metodologia, resultados/discussão e considerações finais ou conclusões). Seis não citam o termo em apenas uma etapa do texto, sendo cinco na metodologia e um nos resultados e discussão. Doze artigos citam o termo em duas partes do texto: cinco na 
introdução e depois nas considerações finais; cinco na introdução e nos resultados e discussão; um nas partes finais do artigo (resultados, discussão e conclusão) e um na metodologia e resultados e discussão. Seis artigos citam o termo em apenas uma parte do artigo (4 em resultados e discussão, 1 na introdução e 1 nas considerações finais/conclusão). O restante dos artigos cita o termo em três momentos (dois citam na metodologia, resultado/discussão e nas considerações finais ou conclusões e quatro citam o termo na introdução, metodologia e resultados e discussão). A figura 2 mostra a distribuição do termo 'Plano de Manejo' nos artigos levantados.

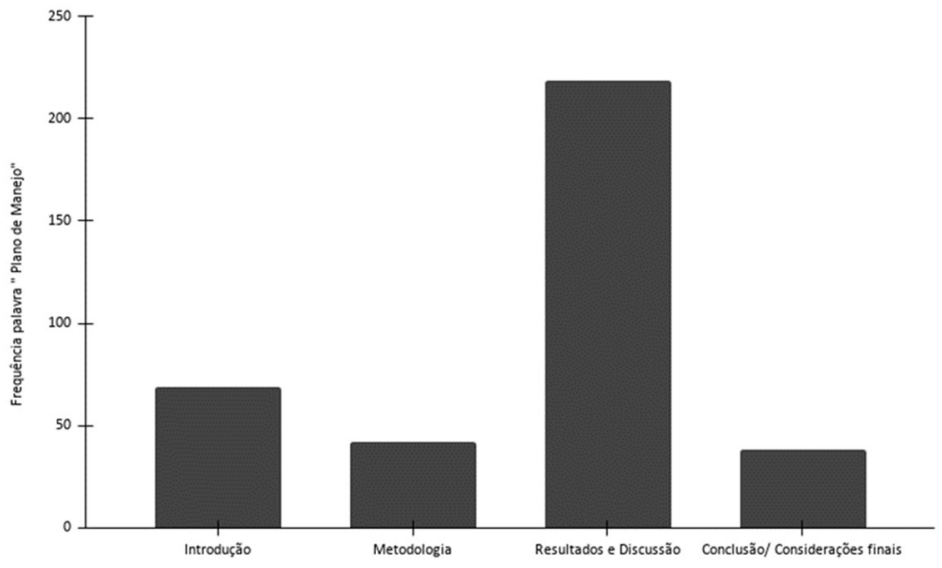

Figura 2: Distribuição da citação do termo ‘Plano de Manejo’ por partes dos artigos.

Dois artigos não citam o termo 'Plano de manejo' em nenhum local do texto exceto pelo resumo. Nestes casos, o termo 'Plano de Manejo' só aparece no texto como forma de justificar o estudo como um potencial suporte científico para alteração ou criação deste documento (ROCHA et al., 2009) ou citam os resultados como possíveis contribuições para a elaboração do Plano de Manejo (MATTOS et al., 2011).

É esperado que o termo 'Plano de Manejo' apareça com maior frequência na parte de resultados e discussão, já que está costuma compor a maior parte dos textos. Porém, é importante avaliar a distribuição do termo no restante da redação. Artigos que o citam no começo, meio e fim do trabalho podem ter contemplado mais este documento em seu processo de pesquisa e análise, enquanto que artigos que o mencionam apenas na introdução ou conclusão podem citá-lo de forma superficial. De fato, todos os artigos que possuem plano de manejo como objeto central do estudo citam o termo em todas as etapas do texto ou em todas exceto uma.

Assim, a busca realizada por este estudo revelou que são menores as investigações que têm o Plano de Manejo com maior ênfase na pesquisa. Apesar de a maior parte dos artigos encontrados indicarem que eles podem contribuir com a elaboração dos Planos de Manejo locais e discutirem sobre as fraquezas dos Planos vigentes nas UCs investigadas, somente $11,11 \%$ destes centram-se em discutir os Planos de Manejo em si, colaborando assim como as UCs em questão. Nesse sentido, verifica-se a distância entre academia e ações sociais (SILVA, 2017). É comum que textos acadêmicos discutam os achados científicos com questões relevantes socialmente, com ocorrências do momento e até que finalizem suas conclusões com palavras de recomendação para as diversas áreas às quais se dedicaram a estudar. No entanto, é imperioso que tais 
ramos trabalhem conjuntamente num esforço de fazer as perguntas certas na averiguação das demandas dos locais e também na busca e aplicação de soluções baseadas em evidências.

Pesquisas científicas que avaliem a qualidade, efetividade e tipos de participação dos atores locais na construção de um documento de gestão tão importante quanto o Plano de Manejo são essenciais para direcionar novas práticas e auxiliar na revisão dos Planos estudados. Além disso, a melhoria do planejamento a longo prazo e o envolvimento efetivo da população do entorno são diretamente relacionados a efetiva conservação ambiental das áreas protegidas (HOCKINGS et al., 2006; IUCN et al., 2019; LOCKWOOD, 2010; MACURA et al., 2015), como as Unidades de Conservação.

\section{CONCLUSÕES}

Nesta revisão bibliográfica focamos na principal forma de comunicação científica, o artigo de revistas e periódicos. Porém, esta não é a única forma que a comunicação entre cientistas pode ser realizada e, tampouco para comunicação com outros profissionais que trabalham na área da conservação ambiental e sustentabilidade. Devido ao caráter recente dos estudos vinculados a governança e gestão é possível que muitos trabalhos sejam realizados nos formatos de dissertações, teses e monografias, ou sejam documentos técnicos ou livros. Futuras revisões devem levar estes componentes em consideração para complementar os resultados deste levantamento. Porém, o baixo número de artigos encontrados em 20 anos de história do Sistema de Unidades de Conservação pode ser um indicativo da necessidade de publicação de resultados revisados por pares para construção do conhecimento dessas áreas, especialmente para avaliação e melhora do Plano Diretor enquanto ferramenta que promova e garanta manejo efetivo e gestão participativa.

Além disso, ressalta-se que a construção do conhecimento sobre as estratégias e ferramentas de gestão e governança das UCs no Brasil, delineadas nos Planos de Manejo, são demandas para a própria revisão e aprimoramento destes documentos e sua consequente prática. Este processo também é essencial no âmbito da comunicação científica entre os pares, que poderão replicar, revisar e contribuir para discussão sobre o tema. Portanto, é necessário que o retorno à comunidade e aos gestores das Unidades de Conservação seja feito não apenas em escala local e como um dever histórico social das universidades, mas em contextos mais amplos.

Há um esforço para diálogo e cooperação mútua entre as instituições e gestores responsáveis pela gestão das UCs e os pesquisadores delas. Tal esforço, no entanto, ainda aparece de maneira modesta nas publicações científicas, o que pode representar fragilidade na execução de ações pautadas em pesquisas e evidências advindas de estudos sobre a efetividade das ações com a finalidade de proteção ambiental e sustentabilidade.

Tendo em vista que a ciência passa por um período de questionamento sobre sua pertinência e veracidade, é urgente que pesquisadores se coloquem à disposição para colaboração real e prática nos locais que Ihes são objeto de estudo. Da mesma forma, faz-se necessário que as comunidades busquem apoio das universidades. 


\section{REFERÊNCIAS}

ABREU, R. S.. Mecanismos de gestão da APA Delta do Parnaíba, Piauí, Brasil. Revista de Geociências do Nordeste, v.2, p.913-920, 2016

ANSILAGO, M.; PEREIRA, N. S.; LEMKE, A. P.; CARVALHO, E. M.. Planejamento de atividades de educação ambiental em uma unidade de conservação sob o olhar de acadêmicos de Gestão Ambiental. RealizAção, v.5, n.10, p.19-26, 2018. DOI: http://doi.org/10.30612/re-ufgd.v5i10.8594

ANTONELLI-PONTI, M.; VALENTI, L.; DÍAZ, D. A. C.; PICOLI, R. M. D. M.; VERSUTI, F. M.. Divulgação científica em psicobiologia: Educação à distância como estratégia para a promoção da extensão universitária. Expressa Extensão, v.23, n.3, p.183-197, 2018. DOI:

http://doi.org/10.15210/ee.v23i3.13668

ARAÚJO-BISSA, C. H.; OLIVEIRA, H. T.. Educação ambiental no Parque Estadual das Fontes do Ipiranga (São Paulo, SP, Brasil): um panorama sobre os programas educativos e sua relação com a Unidade de Conservação. Hoehnea, v.46, n.4, 2019. DOI: http://dx.doi.org/10.1590/2236-8906-22/2019

BAETA, A.; PILÓ, H.. Arqueologia em Unidades de Conservação na Região de Diamantina, MG. As sucessivas ocupações de suas paisagens e cavidades. Revista Espinhaço, p.200-212, 2013. DOI: http://doi.org/10.5281/zenodo.3967934

BARDIN, L.. Análisis de contenido. Akal, 1991.

BEBBER, D. P.; BUTT, N.. Tropical protected areas reduced deforestation carbon emissions by one third from 20002012. Scientific Reports, v.7, n.1, p.1-7, 2017. DOI: http://doi.org/10.1038/s41598-017-14467-w

BERNARDI, I.; SILVA, L. R.; FALCO, P. B.; PIRES, J. S. R.; SANTOS, A. C. A.. Análise comparativa das ferramentas de gestão: Plano de Manejo da APA Itupararanga e os Planos Diretores Municipais. Sociedade \& Natureza, v.32, p.75-91, 2020. DOI: http://doi.org/10.14393/SN-v32-2020-36541

BERTOLIN, C. M.. Desafios na gestão financeira: estudo de caso nas unidades de conservação na Serra de São José, MG. Revista Gestão \& Sustentabilidade Ambiental, v.9, n.1, p.596-616, 2020. DOI: http://dx.doi.org/10.19177/rgsa.v9e12020596-616

BEZERRA, G. C.; CARVALHO, R.; OLIVEIRA, M. C. M.; LYRA, M. R. C. C.. Modelo de excelência em Gestão Pública: o caso da estação ecológica de Caetés. Ambiente \& Sociedade, v.21, 2018a. DOI: http://doi.org/10.1590/18094422asoc0321r3vu18L4AO

BEZERRA, G. C.; OLIVEIRA, R. M. C. M.; LYRA, M. R. C. C.; FRUTUOSO, M. N. M. A.; RODRIGUES, S. S. F. B.. Política Pública, Participação Social e Gestão de Unidades de Conservação: Novos Caminhos para Antigos Desafios. Holos, v.6, p.117-129, 2018b. DOI:

http://doi.org/10.15628/holos.2018.4486

BOLLER, L. L. A; BAZILIO, S.; FIALEK, C. G.. Inventário mastofaunístico da Estação Ecológica Municipal de Boa Ventura de São Roque (PR, Brasil). Acta Biológica Paranaense, v.49, n.1-2, 2020.
BRASIL. Lei federal n. 12.651, de 25 de maio de 2012. Dispõe sobre a proteção da vegetação nativa; altera as Leis nos 6.938, de 31 de agosto de 1981, 9.393, de 19 de dezembro de 1996, e 11.428, de 22 de dezembro de 2006; revoga as Leis nos 4.771, de 15 de setembro de 1965, e 7.754 , de 14 de abril de 1989, e a Medida Provisória no 2.166-67, de 24 de agosto de 2001; e dá outras providências. Brasília: DOU, 2012.

BRASIL. Lei federal n. 9.394, de 20 de dezembro de 1996. Estabelece as diretrizes e bases da educação nacional. Brasília: DOU, 1996.

BRASIL. Lei federal n. 9.985, de 18 de julho de 2000. Regulamenta o art. 225, § 10, incisos I, II, III e VII da Constituição Federal, institui o Sistema Nacional de Unidades de Conservação da Natureza e dá outras providências. Brasília: DOU, 2000.

BRASIL. Ministério do Meio Ambiente. Instituto Chico Mendes de Biodiversidade. Gabinete da Presidência. Instrução Normativa N. 7/2017/GABIN/ICMBIO, de 21 de dezembro de 2017. Estabelece diretrizes e procedimentos para elaboração e revisão de planos de manejo de unidades de conservação da natureza federais. Brasília: DOU, 2017.

BRITO, A. L.. A geodiversidade na Unidade de Conservação do Parque Nacional da Serra do Cipó (MG). Revista Espinhaço, p.25-32, 2015. DOI: http://doi.org/10.5281/zenodo.3962406

BUTCHART, S. H. M.; SCHARLEMANN, J. P. W.; EVANS, M. I.; ARICÒ, S. Q. S.; ARINAITWE, J.; BALMAN, M.; BENNUN, L. A.; BERTZKY B.; BESANÇON, C.; BOUCHER, T. M.; BROOKS, T. M.; BURFIELD, I. J.; BURGESS, N. D.; CHAN, S.; CLAY, R. P.; CROSBY, M. J.; DAVIDSON, N. C.; SILVA, N.; DEVENISH, C.; DUTSON, G. C. L.; FERNÁNDEZ, D. F. D.; FISHPOOL, L. D. C.; FITZGERALD, C.; FOSTER, M.; HEATH, M. F.; HOCKINGS, M.; HOFFMANN, M.; KNOX, D.; LARSEN, F. W.; LAMOREUX, J. F.; LOUCKS, C.; MAY, I.; MILLETT, J.; MOLLOY, D.; MORLING, P.; PARR, M.; RICKETTS, T. H.; SEDDON, N.; SKOLNIK, B.; STUART, S. N.; UPGREN, A.; WOODLEY, S.. Protecting important sites for biodiversity contributes to meeting global conservation targets. PloS One, v.7, n.3, p.e32529, 2012. DOI: http://doi.org/10.1371/journal.pone.0032529

CAMPOS, C. B.; ESTEVES, C. F.; DIAS, D. D. M.; RODRIGUES, F. H. G.. Medium and large sized mammals of the Boqueirão da Onça, North of Bahia State, Brazil. Papéis Avulsos de Zoologia, v.59, 2019. DOI: http://doi.org/10.11606/1807$\underline{0205 / 2019.59 .12}$

CANTO, O.; SOBRINHO, M. V.; VASCONCELLOS, A. M. A.; FERREIRA, L. R.; VASCONCELLOS, A. I.. Conflitos socioambientais e limites da gestão compartilhada em Unidade de Conservação na zona costeira amazônica. Redes (St. Cruz Sul), v.25, n.4, p.1528-1552, 2020. DOI: http://dx.doi.org/10.17058/redes.v25i0.15239

ESTEVES, A. O.; SOUZA, M. P.. Avaliação ambiental estratégica e as áreas de proteção ambiental. Engenharia Sanitária e Ambiental, v.19, p.77-86, 2014. DOI: http://doi.org/10.1590/S1413-41522014019010000443 
FERREIRA, G. H. C.. O Parque Nacional da Serra da Canastra, MG: algumas propostas, conflitos e incertezas territoriais. Revista Cerrados (Unimontes), v.13, n.1, p.111139, 2015.

GELDMANN, J.; COAD, L.; BARNES, M.; CRAIGIE, I. D.; HOCKINGS, M.; KNIGHTS, K.; LEVERINGTON, F.; CUADROS, I. C.; ZAMORA, C.; WOODLEY, S.; BURGESS, N. D.. Changes in protected area management effectiveness over time: A global analysis. Biological Conservation, v.191, p.692-699, 2015. DOI: http://doi.org/10.1016/j.biocon.2015.08.029

GELDMANN, J.; BARNES M.; COAD L.; CRAIGIE I. D.; HOCKINGS, M.; BURGESS N.. Effectiveness of terrestrial protected areas in reducing habitat loss and population declines. Biological Conservation, v.161, p.230-238, 2013. DOI: http://doi.org/10.1016/j.biocon.2013.02.018

GILL, D. A.; MASCIA, M. B.; AHMADIA, G. N.; GLEW, L.; LESTER, S. E.; BARNES, M.; CRAIGIE, I.; DARLING I. E. S.; FREE, C. M.; GELDMANN, J.; HOLST, S.; JENSEN, O. P.; WHITE, A. T.; BASURTO, B.; COAD, L.; GATES, R. D.; GUANNEL, G.; MUMBY, P. J.; THOMAS, H.; WHITMEE, S.; WOODLEY, S.; FOX, H. E.. Capacity shortfalls hinder the performance of marine protected areas globally. Nature, v.543, n.7647, p.665-669, 2017. DOI: http://doi.org/10.1038/nature21708

GONDIM, A. I.; DIAS, T. L. P.; CAMPOS, F. F.; ALONSO, C.; CHRISTOFFERSEN, M. L.. Macrofauna bêntica do Parque Estadual Marinho de Areia Vermelha, Cabedelo, Paraíba, Brasil. Biota Neotropica, v.11, n.2, p.75-86, 2011. DOI: http://doi.org/10.1590/S1676-06032011000200009

GUERRERO, N. R.; TORRES, M.; NEPOMUCENO, I.. Impactos da Lei de Gestão de Florestas Públicas a comunidades tradicionais na Flona do Crepori. Ambiente \& Sociedade, v.23, 2020. DOI: http://doi.org/10.1590/18094422asoc20190054r2vu2020L5AO

HOCKINGS, M.; STOLTON, S.; LEVERINGTON, F.; DUDLEY, N.; COURRAU, J.. Evaluating Effectiveness: A framework for assessing management effectiveness of protected areas. IUCN, 2006.

ICMBio. Monitoramento da visitação em unidades de conservação federais: resultados de 2019 e breve panorama histórico maio 2020. Brasília: ICMBio, 2020.

ICMBio. Roteiro metodológico para elaboração e revisão de planos de manejo das unidades de conservação federais. Brasília, Brasília: ICMBio, 2018.

IUCN; WCPA; ASI. International Union for Conservation of Nature's; World Commission on Protected Areas; Assurance Services International. IUCN Green List of Protected and Conserved Areas: User Manual, Version 1.2. Gland: IUCN, 2019.

KHAN, K. S.; KUNZ, R., KLEIJNEN, J.; ANTES, G.. Five steps to conducting a systematic review. Journal of the Royal Society of Medicine, v.96, n.3, p.118-121, 2003.

KOESTER, A. D.; AZEVEDO, C. R. D.; VOGLIOTTI, A.; DUARTE, J. M. B.. Ocorrência de Atelocynus microtis (Sclater, 1882) na Floresta Nacional do Jamari, estado de Rondônia. Biota Neotropica, v.8, n.4, p.231-234, 2008. DOI: http://doi.org/10.1590/S1676-06032008000400024
LIMA, G. S.; RIBEIRO, G. A.; GONÇALVES, W.. Avaliação da efetividade de manejo das unidades de conservação de proteção integral em Minas Gerais. Revista Árvore, v.29, n.4, p.647-653, 2005. DOI: http://doi.org/10.1590/S0100$\underline{67622005000400017}$

LOCKWOOD, M.. Good governance for terrestrial protected areas: A framework, principles and performance outcomes. Journal of Environmental Management, v.91, n.3, p.754-766, 2010. DOI: http://doi.org/10.1016/j.jenvman.2009.10.005

LOPES, A. L. O.; TRENTIN, G.; SIMON, A. L. H.. Análise da Dinâmica de Coberturas e Usos da Terra como Subsídio ao Planejamento Ambiental de Unidades de Conservação: Aplicações no Parque Estadual do Camaquã (RS-Brasil). Raega-O Espaço Geográfico em Análise, v.46, n.2, p.152170, 2019. DOI:

http://dx.doi.org/10.5380/raega.v46i2.61861

MACE, G. M.. Whose conservation?. Science, v.345, n.6204, p.1558-1560, 2014. DOI:

http://doi.org/10.1126/science.1254704

MACURA, B.; SECCO, L.; PULLIN, A. S.. What evidence exists on the impact of governance type on the conservation effectiveness of forest protected areas? Knowledge base and evidence gaps. Environmental Evidence, v.4, n.1, p.1-29, 2015. DOI: http://doi.org/10.1186/s13750-015-0051-6

MAGANHOTTO, R. F.; SANTOS, L. J. C.; NUCCI, J. C.; LOHMANN, M.; SOUZA, L. C. P.. Unidades de Conservação: limitações e contribuições para a conservação da natureza. Sustentabilidade em Debate, v.5, n.3, 2014. DOI: http://doi.org/10.18472/SustDeb.v5n3.2014

MATTOS, P. P.; NOBRE, I. M.; ALOUFA, M. A. I.. Reserva de desenvolvimento sustentável: avanço na concepção de áreas protegidas?. Sociedade \& Natureza, v.23, n.3, p.409-421, 2011. DOI: http://doi.org/10.1590/S1982$\underline{45132011000300004}$

MEDEIROS, R.; PEREIRA, G. S.. Evolução e implementação dos planos de manejo em parques nacionais no estado do Rio de Janeiro. Revista Árvore, v.35, n.2, p.279-288, 2011.

MESSIAS, M. C. T. B.; SOUSA, H. C. D.; SCALON, V. R.; ROSCHEL, M. B.; CÂNDIDO, E. S.; FUJACO, M. A. G. Phanerogamic flora and vegetation of Itacolomi State Park, Minas Gerais, Brazil. Biota Neotropica, v.17, n.1, 2017. DOI: http://doi.org/10.1590/1676-0611-BN-2016-0236

MEZZOMO, M. M.; GHISSO, K. W.; CAMPOS, D. V.. Caracterização geoecológica como subsídio para estudos ambientais em RPPNs: estudos de casos no Paraná. Revista Árvore, v.38, n.5, p.907-917, 2014. DOI: http://doi.org/10.1590/S0100-67622014000500015

MORAIS, M. S.; GONTIJO, B. M.; MUCIDA, D. P.. Percepções sobre conflitos socioambientais de comunidades do entorno do Parque Estadual do Biribiri, Diamantina, Minas Gerais. Revista Espinhaço, p.2-11, 2018. DOI: http://doi.org/10.5281/zenodo.3952776

NASCIMENTO, L. G. S.; RAMOS, M. A.; ALBUQUERQUE, U. P.; ARAÚJO, E. D. L.. The use of firewood in protected forests: 
collection practices and analysis of legal restrictions to extractivism. Acta Botânica Brasílica, v.33, n.2, p.292-302, 2019. DOI: http://doi.org/10.1590/0102-33062019abb0050

OLDEKOP, J. A.; HOLMES, G.; HARRIS, W. E.; EVANS, K. L.. A global assessment of the social and conservation outcomes of protected areas. Conservation Biology, v.30, n.1, p.133141, 2015. DOI: http://doi.org/10.1111/cobi.12568

RAINHO, A. P.. Desvendando a assimetria entre os programas de verdade na construção do Plano de Manejo: estudo de caso no Parque Nacional de Superagui. Tessituras: Revista de Antropologia e Arqueologia, v.2, n.2, p.269, 2014. DOI: http://doi.org/10.1111/cobi.12568

ROCHA, R. M.; BONNET, N. Y. K.. Ascídias (Tunicata, Ascidiacea) introduzidas no Arquipélago de Alcatrazes, São Paulo. Iheringia, Série Zoologia, v.99, n.1, p.27-35, 2009. DOI: http://doi.org/10.1590/S0073-47212009000100004

SALLUN, F. W.; FERRARI, J. A.; HIRUMA, S. T.; SALLUN, A. E. M.; KARMANN, I.. O carste no plano de manejo do Parque Estadual Intervales e zona de amortecimento, Estado de São Paulo, Brasil. Rem: Revista Escola de Minas, v.63, n.3, p.441448, 2010. DOI: http://doi.org/10.1590/S037044672010000300004

SANTOS, M. P.. Extensão universitária: espaço de aprendizagem profissional e suas relações com o ensino e a pesquisa na educação superior. Revista Conexão UEPG, v.8, n.2, p.154-163, 2012.

SHAMSEER, L.; MOHER, D.; CLARKE, M.; GHERSI, D.; LIBERATI, A.; PETTICREW, M.; SHEKELLE, P.; STEWART, L. A.. Preferred reporting items for systematic review and metaanalysis protocols (PRISMA-P) 2015: elaboration and explanation. Bmj, v.349, 2015. DOI: http://doi.org/10.1136/bmj.g7647

SILVA, L. D.. Para além do Olimpo: por uma divulgação e popularização do conhecimento científico produzido na Universidade Federal do Pará (UFPA). Cadernos de Pesquisa: Pensamento Educacional, Curitiba, v.9, n.23, p.241-264, 2017.

SILVA, R. R. S.. Turismo em Unidade de Conservação: O Caso do Parque Estadual Serra da Baitaca. Turismo-Visão e Ação, v.15, n.3, p.409-418, 2013.

SOUSA, L. M. M.; FIRMINO, C. F.; MARQUES-VIEIRA, C. M. A.; SEVERINO, S. S. P.; PESTANA, H. C. F. C.. Revisões da literatura científica: tipos, métodos e aplicações em enfermagem. Revista Portuguesa de Enfermagem de Reabilitação, v.1, n.1, p.45-54, 2018. DOI: http://doi.org/10.33194/rper.2018.v1.n1.07.4391

SOUZA, A. S.; LIMA, V. R. P.. Conflitos de uso e ameaças a paisagem da Unidade de Conservação Parque Estadual do Poeta e Repentista Juvenal de Oliveira, Campina Grande-PB: uma proposta de intervenção. Revista de Geociências do Nordeste, v.4, p.27-49, 2018.

SOUZA, P. C.; MARTOS, H. L.. Estudo do uso público e análise ambiental das trilhas em uma unidade de conservação de uso sustentável: Floresta Nacional de Ipanema, Iperó-SP. Revista Árvore, v.32, n.1, p.91-100, 2008. DOI: http://doi.org/10.1590/S0100-67622008000100011

VALÉRIO, P. M.; PINHEIRO, L. V. R.. Da comunicação científica à divulgação. Transinformação, v.20, n.2, p.159169, 2008.

VIEIRA, L.; SILVA, F. A. B.; LOUZADA, J.. Dung beetles in a Caatinga Natural Reserve: a threatened Brazilian dry-forest with high biological value. Iheringia, Série Zoologia, v.107, 2017. DOI: http://doi.org/10.1590/1678-4766e2017045

VITALLI, P. L.; ZAKIA, M. J. B.; DURIGAN, G.. Considerações sobre a legislação correlata à zona-tampão de unidades de conservação no Brasil. Ambiente \& Sociedade, v.12, n.1, p.67-82, 2009. DOI: http://doi.org/10.1590/S1414$\underline{753 \times 2009000100006}$

WAGAR, J. A.. Recreational carrying capacity reconsidered. Journal of Forestry, v.72, n.5, p.274-278, 1974. DOI: http://doi.org/10.1093/jof/72.5.274

WATSON, J. E. M.; DUDLEY, N.; SEGAN, D. B.; HOCKINGS, M. The performance and potential of protected areas. Nature, v.515, n.7525, p.67-73, 2014. DOI: http://doi.org/10.1038/nature13947

A CBPC - Companhia Brasileira de Produção Científica (CNPJ: 11.221.422/0001-03) detém os direitos materiais desta publicação. Os direitos referem-se à publicação do trabalho em qualquer parte do mundo, incluindo os direitos às renovações, expansões e disseminações da contribuição, bem como outros direitos subsidiários. Todos os trabalhos publicados eletronicamente poderão posteriormente ser publicados em coletâneas impressas sob coordenação da Sustenere Publishing, da Companhia Brasileira de Produção Científica e seus parceiros autorizados. Os (as) autores (as) preservam os direitos autorais, mas não têm permissão para a publicação da contribuição em outro meio, impresso ou digital, em português ou em tradução. 